\title{
The stellar populations of the AGN/Starburst galaxy NGC7582
}

\author{
T. V. Ricci ${ }^{1 \dagger}$, J. E. Steiner ${ }^{1}$, R. B. Menezes $^{1}$, A. Garcia-Rissmann ${ }^{2}$ \\ and R. Cid Fernandes ${ }^{3}$ \\ ${ }^{1}$ Instituto de Astronomia, Geofísica e Ciências Atmosféricas, Universidade de São Paulo, \\ Rua do Matão, 1226, São Paulo - SP, Brasil \\ ${ }^{2}$ Gemini Observatory \\ ${ }^{3}$ Universidade Federal de Santa Catarina \\ ${ }^{\dagger}$ tiago@astro.iag.usp.br
}

\begin{abstract}
NGC 7582 is defined as a Starburst/AGN galaxy, since its optical and X-Ray spectra reveal both characteristics. In this work, we show the results of a stellar population modeling in a datacube taken with the Gemini South telescope. We found that $\sim 90 \%$ of the light in the field of view is emitted by stars that are less than 1 billion years old. A strong burst occurred about $\sim 6$ million years ago and has nearly solar metallicity. We also found a Wolf-Rayet cluster.
\end{abstract}

Keywords. Active galactic nuclei; Starburst galaxies; population synthesis; spectroscopy.

\section{Introduction}

Starburst galaxies are characterized by their strong and narrow emission lines in the optical spectra, due to a strong star formation in the galaxy center. Seyfert galaxies have broader emission lines and are interpreted as being produced by photoionization by a power-law continuum associated to a supermassive black hole. NGC 7582 has a typical H II region spectrum in the optical (Veron et al. 1981) but its X-Ray emission is typical of a Seyfert 1 galaxy (Ward et al. 1978). In this work our goal is to analyze the stellar population located in the 3.5 " x 5 " central region of NGC 7582 .

\section{Observations and Methodology}

Our observations were taken with the GMOS-IFU (Allington-Smith et al. 2002) on the Gemini South telescope. We used the B600-G5323 grating, producing a spectral range of $4230-7070 \dot{A}$ with $\mathrm{R}=2400$ (as measured from the $5577 \dot{A}$ sky line). The final datacubes are corrected from the atmospheric differential refraction; we also performed a Richardson-Lucy deconvolution with 6 iterations on the image in each spectral pixel. The stellar population synthesis was made using the Starlight code (Cid Fernandes et al. 2005) on the spectrum of each spatial pixel, with a sampling of 0.05 ” x 0.05 ". Maps of different population were constructed, revealing age and metallicity structures. These results will be published elsewhere.

\section{Results and conclusions}

Figure 1 shows the cumulative flux fraction as a function of stellar age. It is clear that about $90 \%$ of the light in the field of view is emitted by stars that are less than 1 billion 


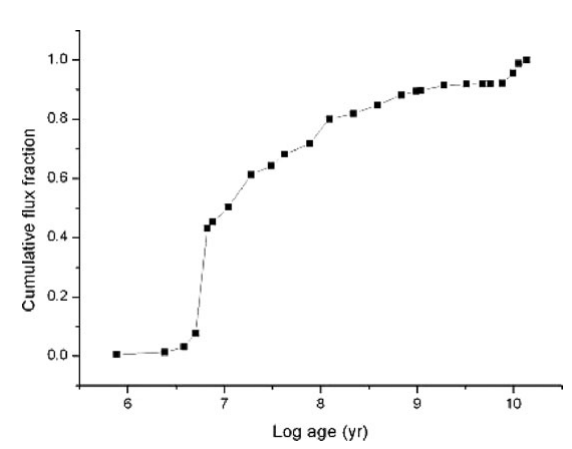

Figure 1. Cumulative flux fraction as a function of the stellar age of the entire field of view.

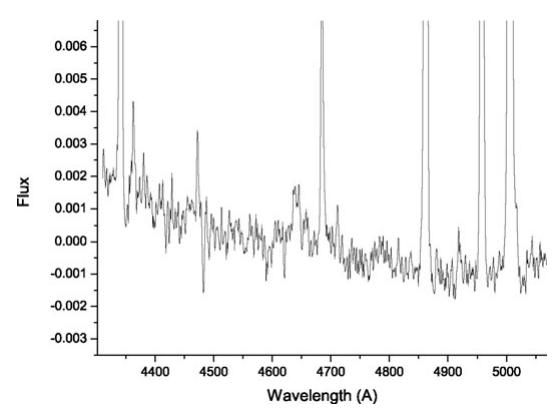

Figure 2. The spectrum showing the WR feature at 4600 to $4700 \dot{A}$. This spectrum was extracted at a position 1.4" west and 1.0 " north of the AGN.

years old. There seems that a more or less continuum star formation episodes have occurred in this period. Nearly half of the light is emitted by stars less than 10 million years old. A strong event occurred $\sim 6$ million years ago. This young stellar population shows nearly solar metallicity and is concentrated in three clumps that might be associated with the ionizing sources of the three observed H II regions. One of the clumps, located nearly 1.4" West and 1.0" North of the AGN presents a Wolf-Rayet feature, shown in Figure 2. This feature has broad C III/N III + He II emission between $4600 \dot{A}$ and $4700 \dot{A}$.

I would like to thank FAPESP for the financial support.

\section{References}

Allington-Smith, J. et al. 2002, PASP, 114, 892

Cid Fernandes, R., Mateus, A., Sodré, L., Stasińska, G., \& Gomes, J. M. 2005, MNRAS, 358, 363

Veron, P., Veron, M. P., Bergeron, J., \& Zuidervijk, E. J. 1981, A\&A, 97, 71

Ward, M. J., Wilson, A. S., Penston, M. V., Elvis, M., Maccacaro, T., \& Tritton, K. P. 1978, ApJ, 223, 788 